\title{
Studying 2D Materials by Means of Microscopy and Spectroscopy with Low Energy Electrons
}

\author{
Ivo Konvalina ${ }^{1 *}$, Benjamin Daniel ${ }^{1}$, Martin Zouhar $^{1}$, Jakub Piňos ${ }^{1}$, Tomáš Radlička ${ }^{1}$, Luděk Frank ${ }^{1}$, \\ Ilona Müllerová ${ }^{1}$ and Eliška Materna Mikmeková ${ }^{1}$ \\ 1. Department of Electron Microscopy, Institute of Scientific Instruments of the CAS, v. v. i., Brno, \\ Czech Republic. \\ * Corresponding author: konvalina@isibrno.cz
}

Two-dimensional (2D) materials have recently become very popular because of their interesting properties and rich application potential [1]. Progress in production of new high quality 2D materials necessitates to study and analyze these materials. Such studies can be performed via scanning electron microscopy with slow and very slow electrons and time-of-flight (ToF) spectroscopy.

In order to gain a better understanding of contrast formation in the energy range below $100 \mathrm{eV}$, more general knowledge about the interaction of (very) low energy electrons with solids is required. The necessary information will be provided with a newly developed ultra-high vacuum (UHV) scanning low energy electron microscope (SLEEM) which includes several enhancements compared to commercially available devices.

Our apparatus (Figure 1,2) can operate as a standard scanning electron microscope (SEM) which allows to make an image of the sample. The microscope is equipped with an electron gun with $5 \mathrm{keV}$ primary beam energy developed by Delong Instruments [2], an in-house build specimen stage with biased specimen holder for use of the cathode lens mode in the SLEEM [3], and several electron detectors.

A newly added operation mode allows to measure the ToF spectra [4] of electrons transmitted through a sample. In order to implement the ToF spectrometer functionality, the microscope has to work in a pulse mode. Because of that, the commercial electronics provided with the multi-channel plate (MCP) detector [5] has been complemented by a pulse generator deflecting the primary electron beam. The software supplied to the MCP detector, integrated in the ToF spectrometer, records the timestamps of detections for each electron pulse sent towards a given pixel of the sample.

Simulated ray tracing of transmitted electrons (TEs) in the region between the sample and MCP detector (Figure 3) allows to determine optimal parameters of the spectrometer. A single measurement of the TEs detected by the ToF spectrometer produces a - time domain - ToF spectrum (Figure 4) that will be converted to the energy domain thus yielding an energy loss spectrum. This energy domain spectrum will provide an input to study of the electron scattering phenomena in the $2 \mathrm{D}$ materials at very low kinetic energies. A crucial parameter describing the electron scattering is the inelastic mean free path [6] which can be extracted from the energy loss spectrum.

References:

[1] GR Bhimanapati et al., ACS Nano 9 (2015), p. 11509-11539.

[2] DIGUN, https://www.delong.cz/products/electron-guns/ (accessed February 18, 2019).

[3] I Müllerová and L Frank, Adv. Imag. Elect. Phys. 128 (2003), p. 309-443. 
[4] WE Stephens, Phys. Rev. 69 (1946), p. 691.

[5] RoentDek Delayline Detectors, www.roentdek.com/detectors/ (accessed February 18, 2019).

[6] MP Seah and WA Dench, Surf. Interface Anal. 1 (1979) p. 2-11.

[7] The authors acknowledge funding from the Technology Agency of the Czech Republic (Centre of Electron and Photonic Optics, no: TN01000008).

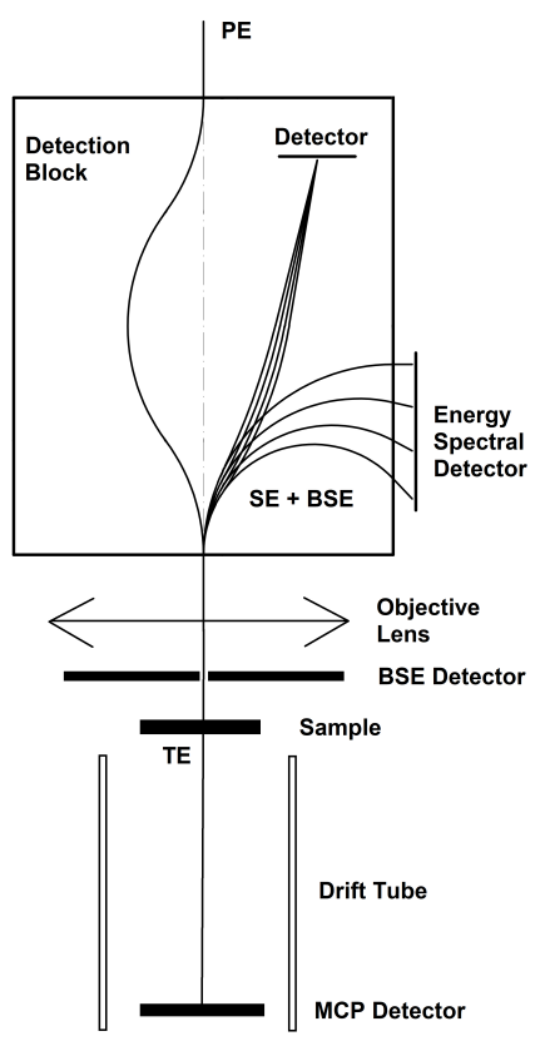

Figure 1. Schema of the UHV SLEEM system.

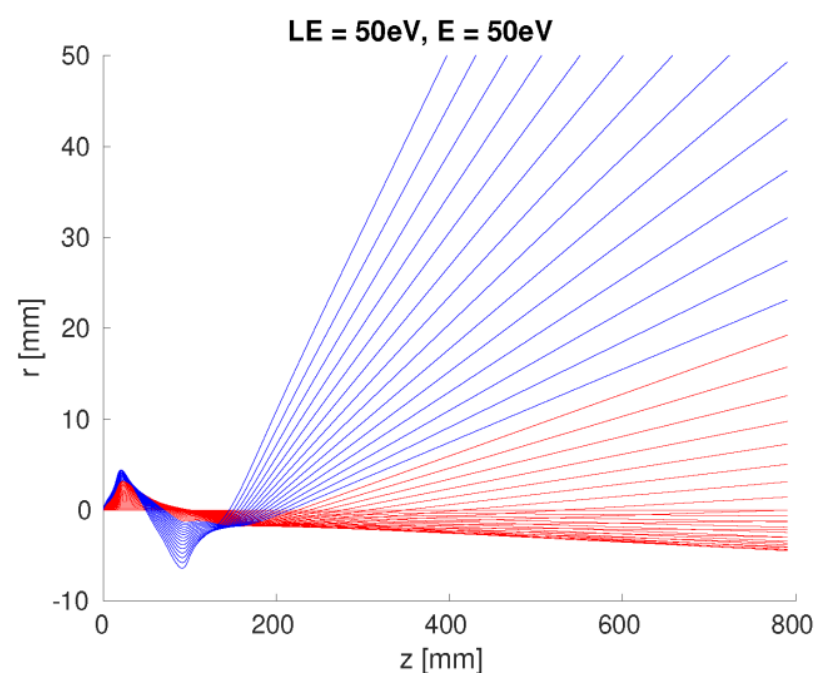

Figure 3. Simulation of transmitted electron trajectories for landing energy of $50 \mathrm{eV}$.

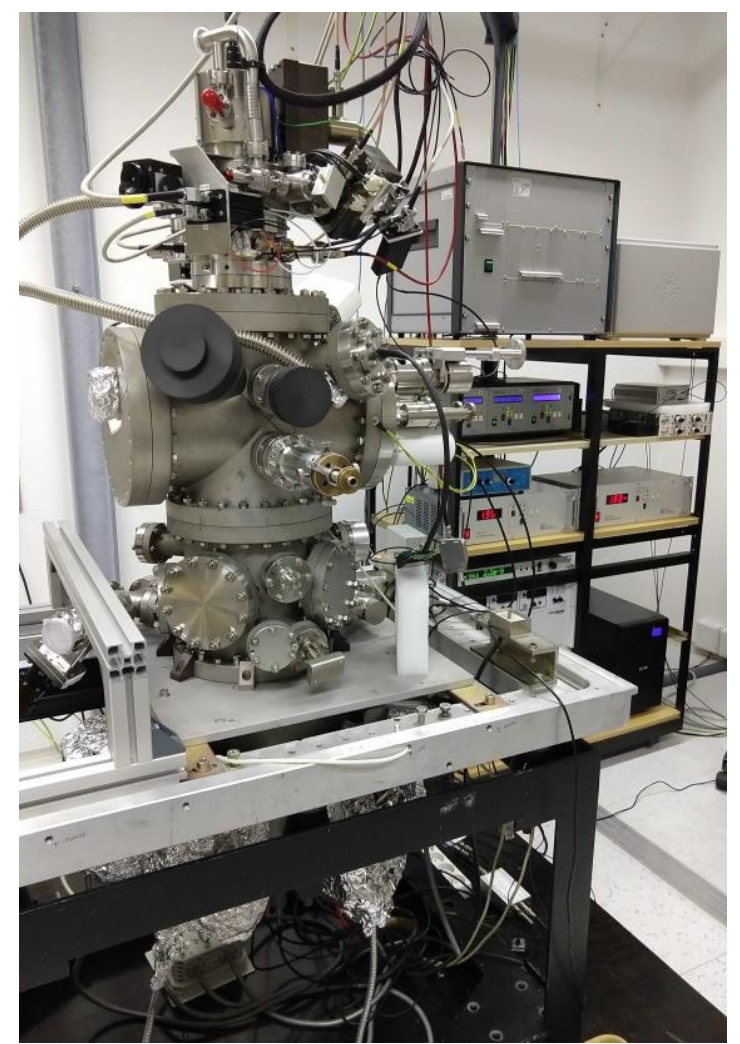

Figure 2. View of the UHV SLEEM system.

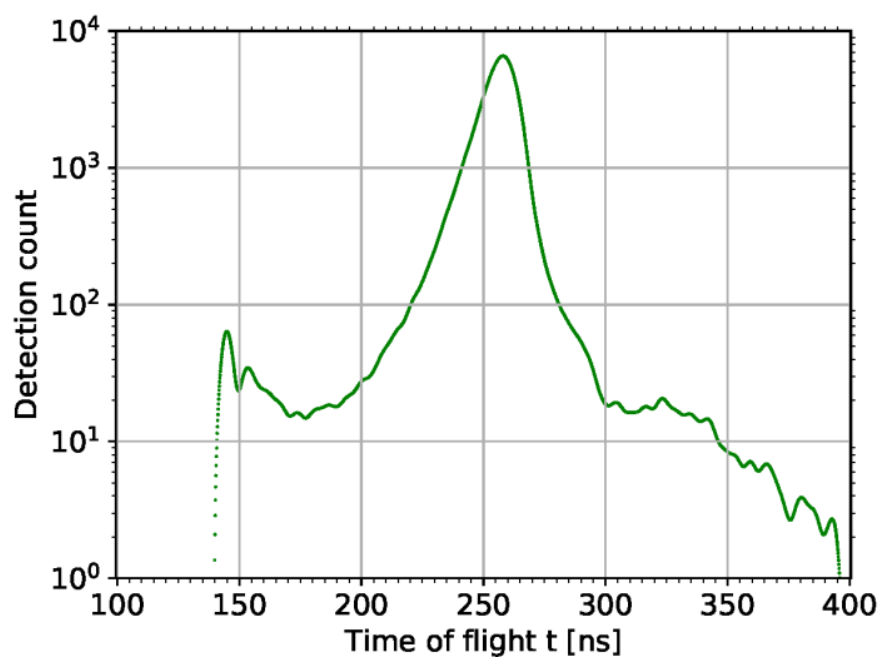

Figure 4. ToF spectrum for one layered graphene. 